\title{
A Study of Routing Protocols in VANETs
}

\author{
Rupinder Kaur ${ }^{1}$, Dr. Gagandeep Jagdev ${ }^{2}$ \\ ${ }^{1}$ Research Scholar (M.Tech.), Yadavindra College of Engineering, Talwandi Sabo (PB) \\ ${ }^{2}$ Dept. of Comp. Science, Punjabi University Guru Kashi College, Damdama Sahib (PB)
}

\begin{abstract}
A new wireless technology which enables vehicles to communicate with each other is being developed and is referred as Vehicular Ad hoc Networks (VANETs). These could enable a number of innovative schemes to reduce traffic congestion and greenhouse gas emissions. VANETs are exceptional kind of Mobile Ad hoc Networks (MANETS). Individual vehicles act as nodes in VANETs. Messages are passed by multi-hop communication in VANETs. Mostly VANETs make use of Wi-Fi for communication although cellular and satellite are also being looked at for communications. Because of high mobility speed of nodes in VANETs which can range up to $240 \mathrm{~km} / \mathrm{hour}$, VANETs use special Wi-Fi protocol IEEE 802.11p. VANETs are not true MANETs as they rely on some kind of infrastructure. The infrastructure comprises of Road Side Units (RSUs) which is responsible for connecting the network to the internet and databases containing traffic information and help overcome network partitions. Vehicles in VANETs can have relatively predictable mobility if they stick to roads, obey speed limits, stop at traffic lights etc. In this research paper, the central theme is analyzing the working of VANETs, routing protocols involved in VANETs and discussing challenges faced by VANETs.
\end{abstract}

Keywords: MANETs, Routing protocols, RSUs, VANETs, Vehicle-to-Vehicle (V2V), Vehicle-to-roadsideInfrastructure (V2X).

Abbreviations: MANETs, RSU, VANETs, V2V, V2X

\section{INTRODUCTION}

The technology using vehicles as nodes to form a mobile ad hoc network for diffusion of safety and entertainment messages is referred as Vehicular Ad hoc Networks (VANETs). Figure 1 shows the working of VANETs.

Driving refers to the regular change of location. This refers to continuous demand related to information of current location and data concerning surrounding traffic, routes and much more. The important category in this regard is that of driver assistance and car safety. This involves information received from sensor data from other cars. A driver can receive information which can be classified in different categories mentioned as under [1].

$>$ Related to road condition and maintenance, brake warning delivered by preceding car, tailgate and collision warning, weather forecast, forewarning of traffic jams, carefulness to an accident behind the next bend and many other such information.

$>$ Second category is of infotainment which comprises internet access, chatting and interactive games between vehicles close to each other.

$>$ Third category is related to local information as free parking space, information related to fuel prices, tourist information, information regarding nearby service station.

$>$ Fourth category is related to maintenance of vehicles. For example, online help received from your vehicle mechanic when it breaks down or service information of vehicle. 


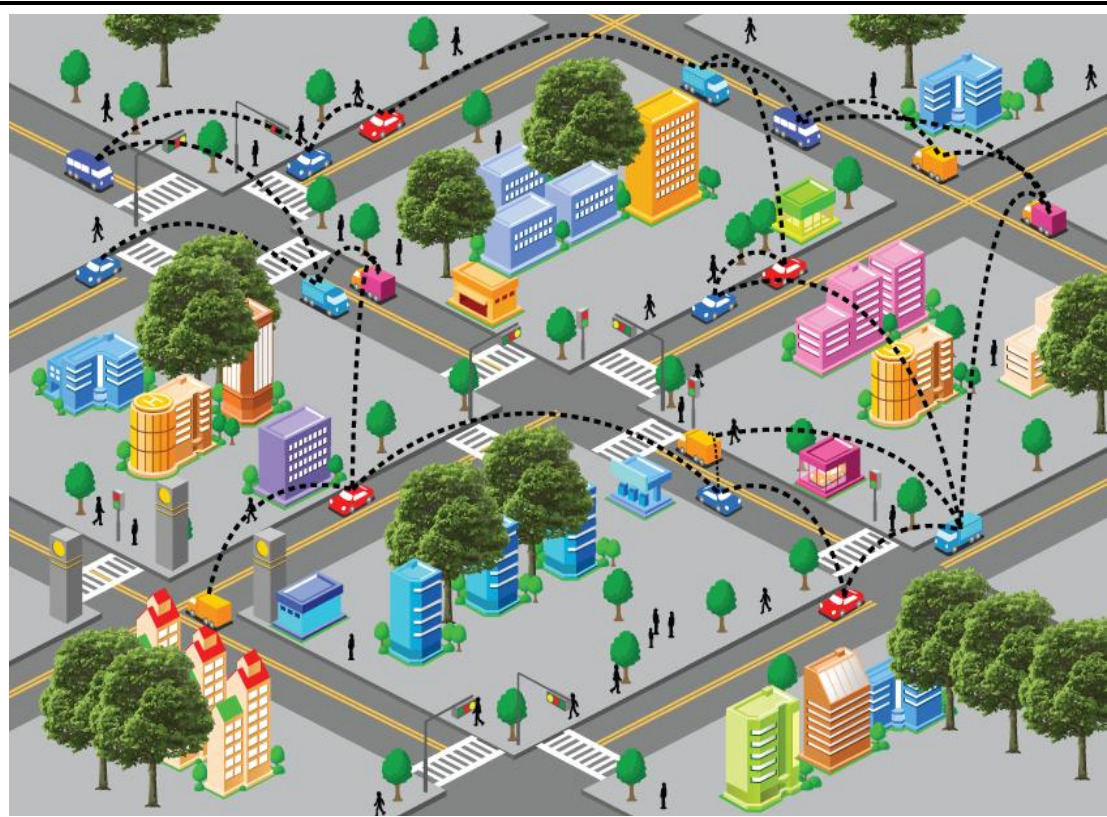

Figure1. Working of VANETs

Unlike cellular networks, VANETs conduct communication without any kind of help from traditional infrastructure. VANETs are classified into two main categories mentioned as under (Figure 2).

- Vehicle-to-roadside-infrastructure (V2X) -In this category communication is established between vehicles and roadside units like traffic lights and road lights, via internet access.

- Vehicle-to-Vehicle (V2V) - In this category, vehicles themselves organizes the network and share information with no central control.

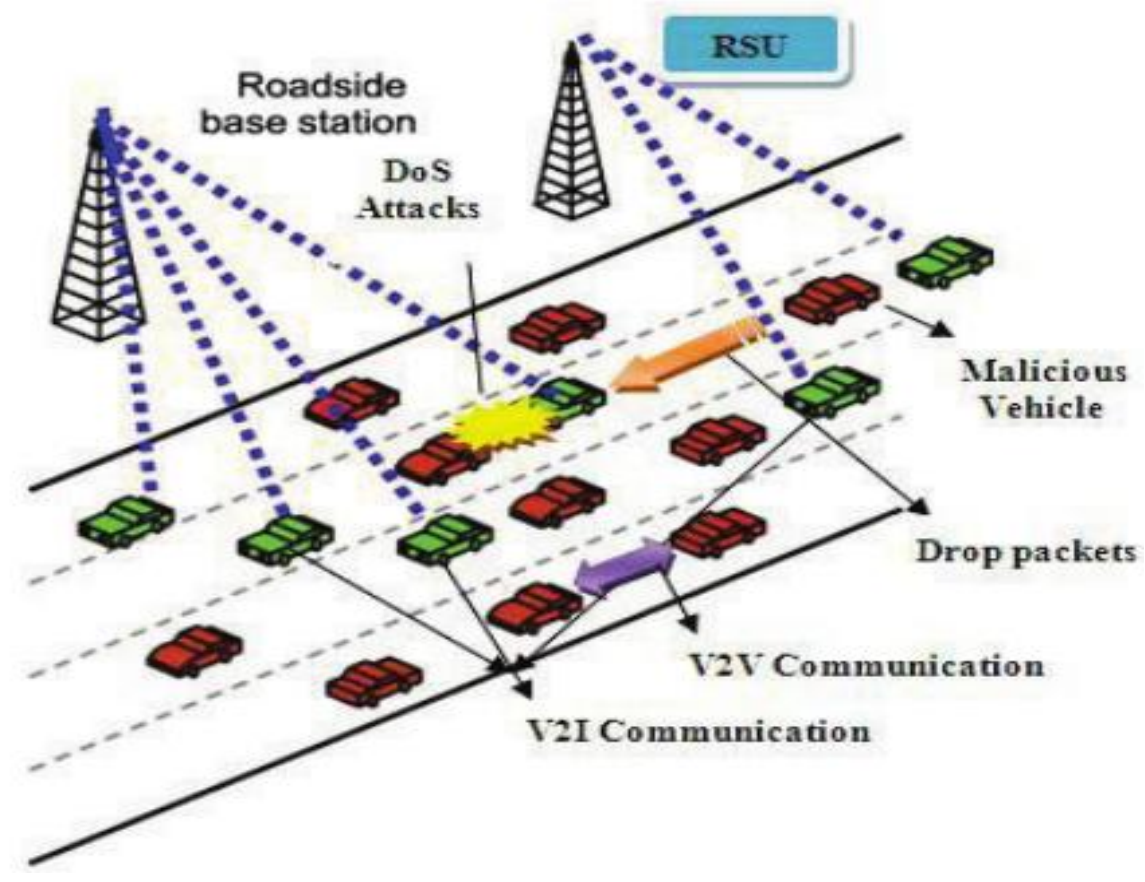

Figure2. Working of $V 2 X$ and $V 2 V$

Based on type of information provided, VANET applications can be categorized in two different categories mentioned as under [2].

$>$ Safety

It includes information concerning traffic conditions.

- Traffic collisions

- Congestion 
- Emergency vehicle warnings

- Overtaking vehicle warnings

- Lane changing assistance

- Pre-accident warnings.

$>$ Infotainment

Infotainment is combination of information and entertainment and provides drivers with local services like

- Internet access

- Media downloads

- Point of interest notification.

\section{Features OF VANETS}

VANETs is a branch of Mobile Ad-hoc Networks (MANETs). The nodes in case of VANETs are vehicles on road. The prominent features of VANETs are mentioned as under [3].

\section{High Dynamic and Frequent Disconnection}

The duration time of connection in VANETs is much less than MANETs because of the high speed of nodes or vehicles involved in VANETs. This results in frequent breakage of links. Normally road range is assumed to be $240 \mathrm{~m}$ and the gap between two vehicles moving in the same direction is $10 \mathrm{mph}$, for example, $50 \mathrm{mph}$ and $60 \mathrm{mph}$, so the maximum connection time is 107 seconds if the faster vehicle is catching the slower one from the very beginning. But if same vehicles under consideration are moving in opposite direction, the maximum connection time is less than 10 seconds. But when we consider MANETs with speed of $1 \mathrm{~m} / \mathrm{s}$ between two nodes, the connection duration can be up to 480 seconds which is approximately 4 times of 109 seconds. Repeated disconnection of links in VANETs also causes a frequent change in network topology.

\section{Density Variation}

Due to the high mobility of nodes (vehicles) in VANETs, the density of vehicles varies according to road condition. Road gets congested to varying degrees during rush hours and such conditions often result in traffic jams. Urban areas have a high density of vehicles as compared to rural areas or highways. The vehicle density is closely related to network connectivity and effects the performance of communication schemes dedicated for VANETs.

\section{Regular Trace}

In MANETs nodes are free to move in any direction along an irregular trace. But in VANETs, due to confinement of roads, bridges, and buildings, the traces of nodes are found to be regular along the road. The traces of nodes are not completely independent in VANETs as the speed of the vehicle is influenced by the speed of surrounding vehicles on road.

\section{Power Consumption}

In MANETs, power consumption has always been an issue as the carrier is a tiny device and the battery available for making sensors operational is limited. But in VANETs, the power is supplied by an onboard source in the form of vehicle battery which provides enough energy for VANETs sensors and power remains not an issue anymore.

\section{$>$ Varying Environment for Communication}

Two types of environments are considered in VANETs, urban scenarios, and highway scenario. In the urban scenario, the movement of vehicles is slow because of complicated traffic patterns, congestion on roads and traffic lights. The buildings and traffic lights form a vehicle-to-infrastructure network and block radio propagation. Comparatively, conditions are simpler in highway scenario. Vehicles move at higher speeds, roads are straight paths and there's less interference from roadside units. In highway scenario, communication is in the form of vehicle-to-vehicle. 


\section{Routing Protocols in Vanets}

Because of similarity between MANETs and VANETs, the protocols used in MANETs can also be applied in VANETs. But the performance of these protocols varies a lot in both. The two key factors to be considered in protocol design of VANETs are those of reliability and stability. Routing protocols in VANETs can be categorized as shown in Figure 3.

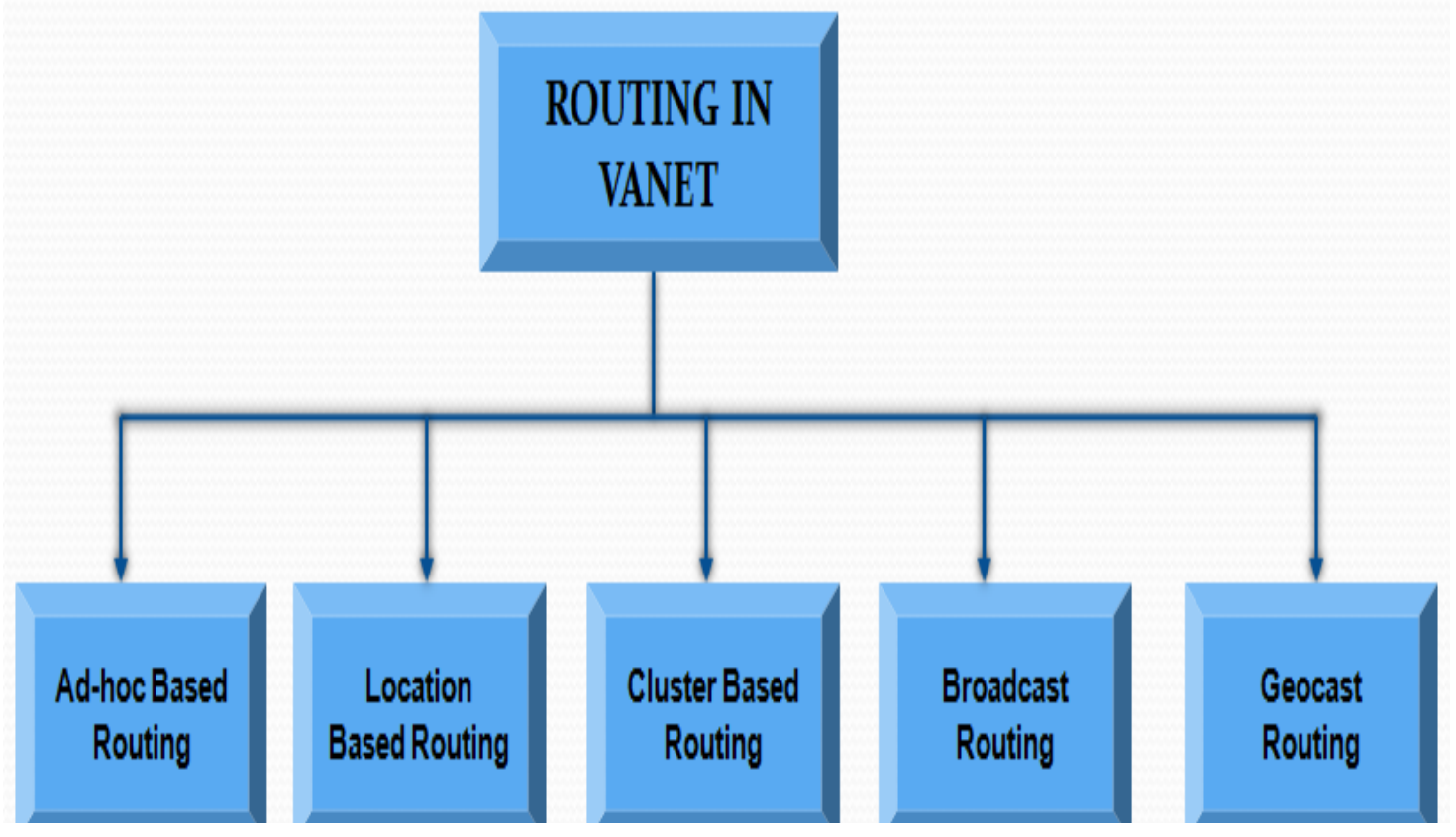

Figure3. The figure depicts different types of routing in VANETs

\section{Broadcast Routing}

\section{Flooding Broadcast}

Flooding broadcast is the simplest way of message broadcasting. In this scheme, every node which receives the message forwards it. Problems related to this scheme are a waste of bandwidth and repeated collisions. Broadcast storm in VANETs is quantified. Therefore, the transmission performance is significantly affected by the increase in vehicle density with the increase in the number of hops, total delay and packet loss ratio. Different selective forwarding schemes are brought into use to improve the performance of flooding scheme.

- In weighted p-persistence broadcasting, the first time received message is forwarded by the receiver with specific probability $\mathrm{p}$ (ratio of the distance between the transmitter and the receiver to the transmission range).

- Slotted 1-Persistence allows the receiver to forward the message with probability 1 in the priory assigned time slot. The assigned time slot is related to one-hop delay, distance between transmitter and receiver and the total number of time slots.

- Slotted p-persistence is the hybrid scheme in which receivers forward the message with aspecific probability in priory assigned time slot. Both the probability and assigned time slot is related to the distance between transmitter and receiver. Packet loss ratio and broadcast redundancy are reduced up to $70 \%$ when compared with flooding and propagation latency which is still at acceptable levels.

\section{Border Node-Based Routing (BBR)}

BBR protocol is meant for the partially connected network. For example, VANETs in the rural area. The border node isone with the minimum number of common neighbor nodes which is the furthest node from the transmitter within the transmission range. In Figure 4, the common neighbors between vehiclesTx and 1 are vehicle 4 and 5. While in case of vehicle 2, 3, 4 and 5, the number of common vehicles with vehicle Tx is 3 or more. Therefore, vehicle 1 is border node. As the message is broadcasted by the transmitter, the border node of the transmitter forwards the message in flooding way [4]. 


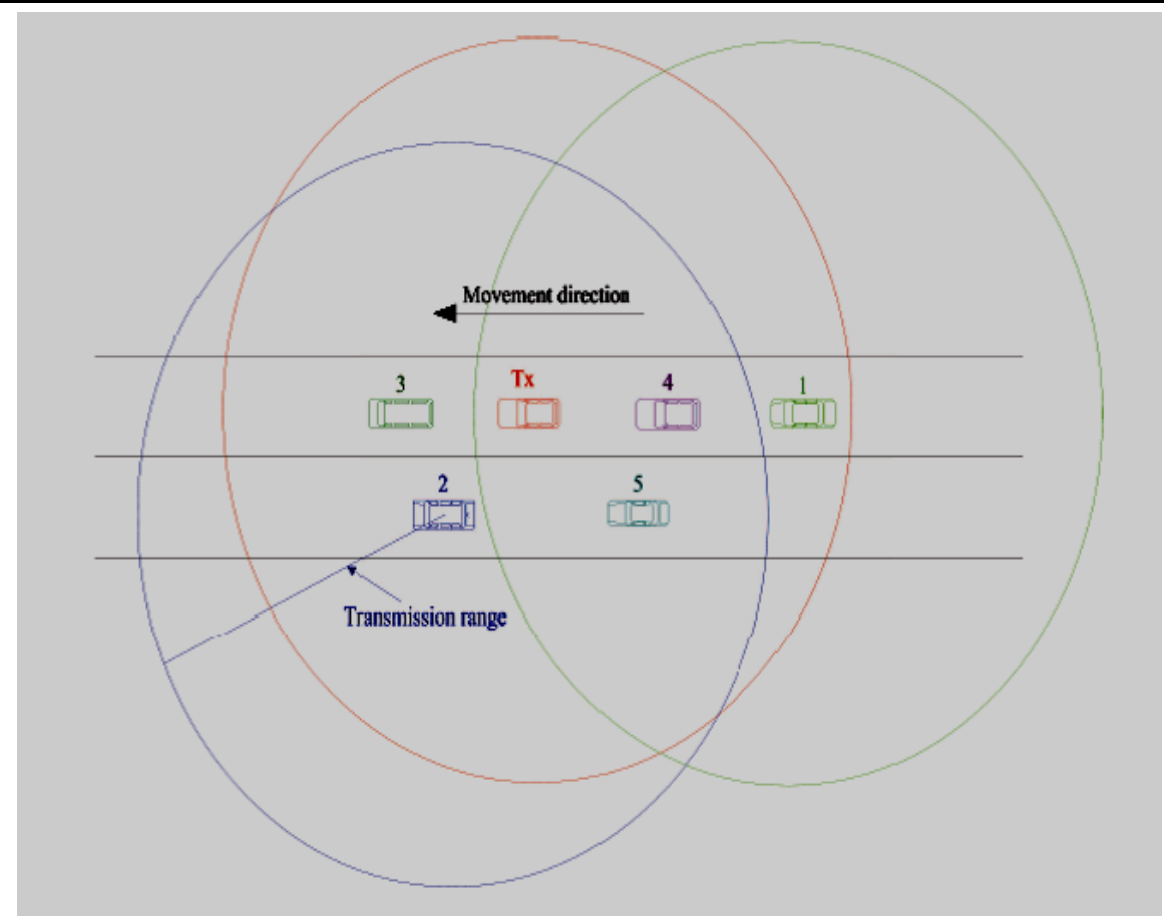

Figure4. The figure illustrates the working of BBR

\section{Cluster-Based Protocols}

In this category, message propagation is conducted by a virtual chain of networks. Vehicles are grouped geographically as clusters as shown in Figure 5. Radio range of wireless devices decides the cluster size to be adopted in the network. Each cluster has a cluster head $(\mathrm{CH})$ and takes charge of inter-cluster communication. Cluster members in the same cluster communicate with each other directly and via cluster head $(\mathrm{CH})$ with vehicles in other clusters. The reliable communication between the cluster heads affects the performance of the system. In the cross-layer protocol, predefined backbone members $(\mathrm{BM})$ are selected to forward messages prior to the propagation and are referred as Dynamic Backbone Assistance MAC (DBA-MAC). If a vehicle has not received any messages from nearby BMs for a specific time period, it upgrades itself as a BM and broadcasts an inspiration to create BM network. The suitability as a BMis measured by the relative distancebetween the $\mathrm{BM}$ candidates and the BM at the end of the predefined period, the one with thelongest distance winning contention with the highest probability. While message propagation is on, only BMs forward messages from preceding BM. If at some time, BM fails to forward a message, all other vehicles start an argument to forward the message.

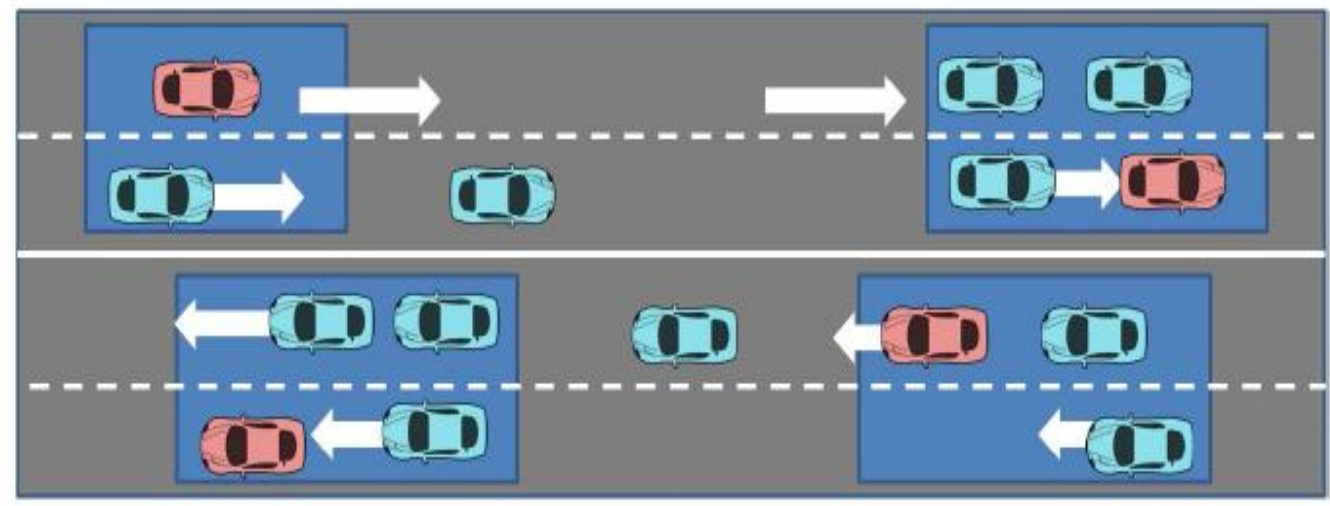

Cluster

Cluster Head Vehicle

Figure5. Working of Cluster-based routing protocols 


\section{Ad Hoc (Topology-Based) Routing Protocols}

Like MANETs, Ad hoc On-Demand Vector (AODV) and Dynamic Source Routing (DSR) are also applicable in VANETs as shown in Figure 6. It is assumed that vehicles are equipped with GPS devices which provide the location and speed of vehicles exactly [6]. In AODV, the source node broadcasts a route request (RREQ) before the message is actually transmitted. The nearby node responds to this message with a route reply (RREP), if it stores a route to the destination and if not, the node rebroadcasts the RREQ. At some time, when the destination receives RREQ, it responds with RREP and RREP is propagated back to the source. The message transmission begins as soon as RREP is received. In case if a RREP with less hop count to the destination is received, the route is updated by the source and a new route is adopted for message propagation. In case if a link failure occurs, the upstream node of the breakpoint reports to the source and source re-initiates finding a route if necessary. DAODV further improves AODV by introducing mobility information in route selection. It is supposed that every vehicle in the network is fortified with Global Positioning System (GPS) and the mobility information. During route discovery, the direction of candidates needs to be checked. All the candidates moving in the same direction as source and destination are referred as potential candidates. The potential candidates within the source and destination are only responsible for responding to the source. If there is no potential candidate between source and destination, all potential candidates respond to the source to build the route.

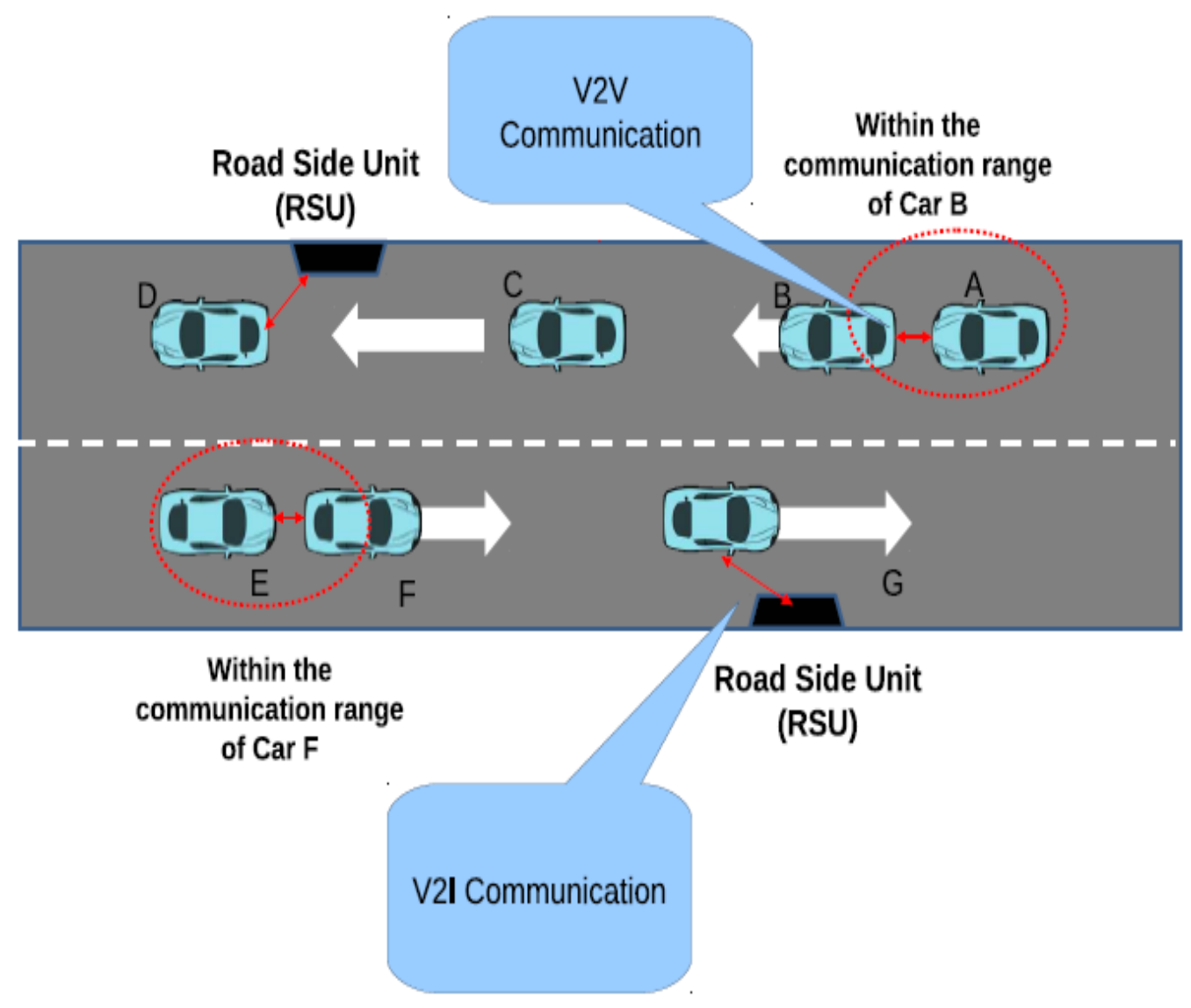

Figure6. Working of Ad hoc based routing protocols in VANETs

\section{Location Based Protocols}

Global Positioning System (GPS) and route map provide the location of vehicles in VANETs. Location information can be used to find a route from source to the destination for propagating the message. The traffic-related information is often used to forecast the location of possible relays for the stability of the route $[5,7]$.

- Greedy Perimeter Stateless Routing (GPSR) is the most popular location-based protocol (Figure 7). In this, it is expected that the position of every node and destination within the network is 
known by all others. As the message is transmitted, it finds the node closest to the destination to forward the message. If in case, transmitter fails to find the greedy path, the perimeter mode is used to forward the packet in which it traverses along the closer faces of a planar graph of the network and greedy forwarding restarts if the node closer to the destination is found as compared to the current node. This procedure comes to a halt when a node closer to the destination is found and greedy forwarding procedure continues. In VANETs GPSR has to handle the mobility of the nodes. When the node is about to transmit a message, it calculates the geographic location of each neighbor after a specific time $\mathrm{T}$, based on current location, the direction of movement and current velocity. Among the left behind nodes, the one closest to the destination implements forwarding the message.

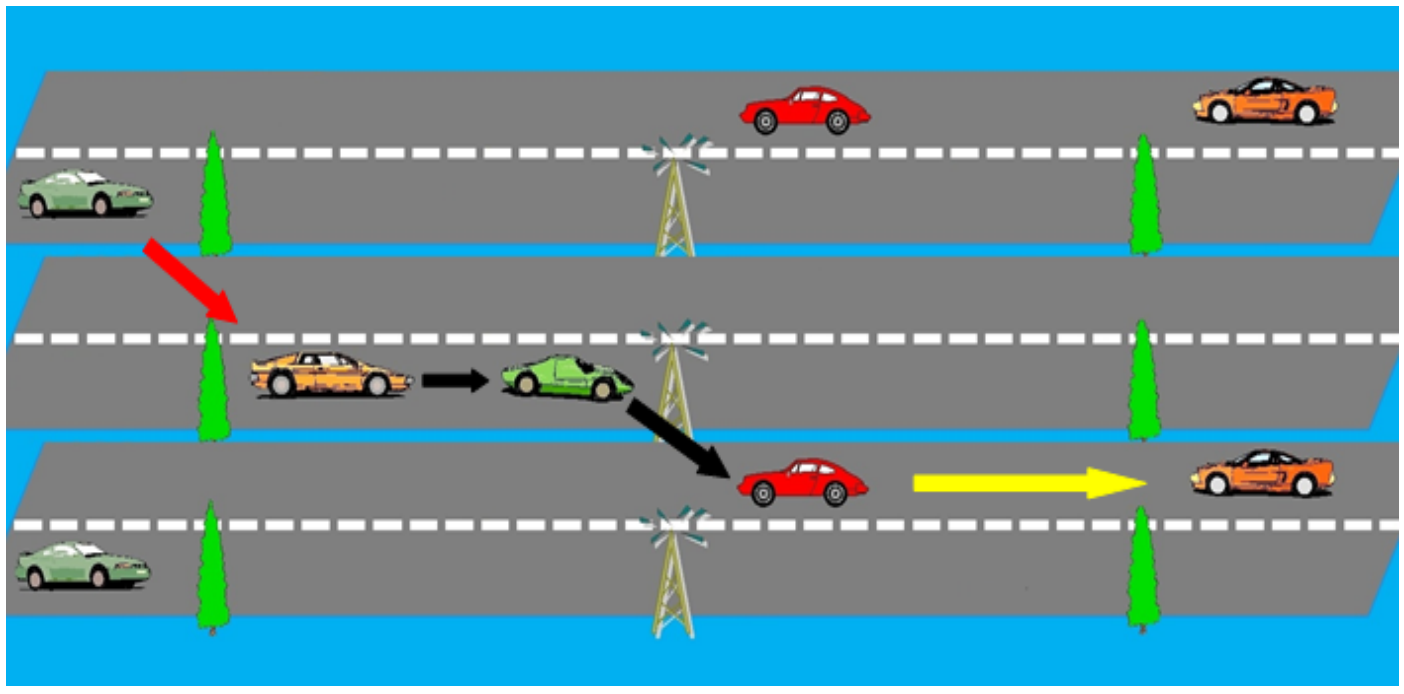

Figure7. The figure shows the working of GPSR

\section{Geo Cast Protocols}

Geocast routing protocols set a Region of Interest (ROI) which is determined by the location of the message source. Packets are sent to all the vehicles within ROI, for example, information on accidents and congestion (Figure 8). Vehicles travelling in opposite direction may be used to relay the message through the ROI. On the other hand, the vehicles travelling in the same direction, are grouped based on transmission range and a group head $(\mathrm{GH})$ is selected for every individual group. Once an incident begins, the vehicle which is part of the incident broadcasts a message to notify other vehicles about the incident. In case if network condition fails to guarantee that the message is transmitted throughout the ROI, as in the disconnected case, GHs travelling in opposite direction take charge of forwarding the message. Once a $\mathrm{GH}$ receives the message and rebroadcasts it, the source stops periodical broadcast upon sensing the rebroadcasting. The GH travelling in the opposite direction rebroadcasts the message before itmoves out of the region of interest.

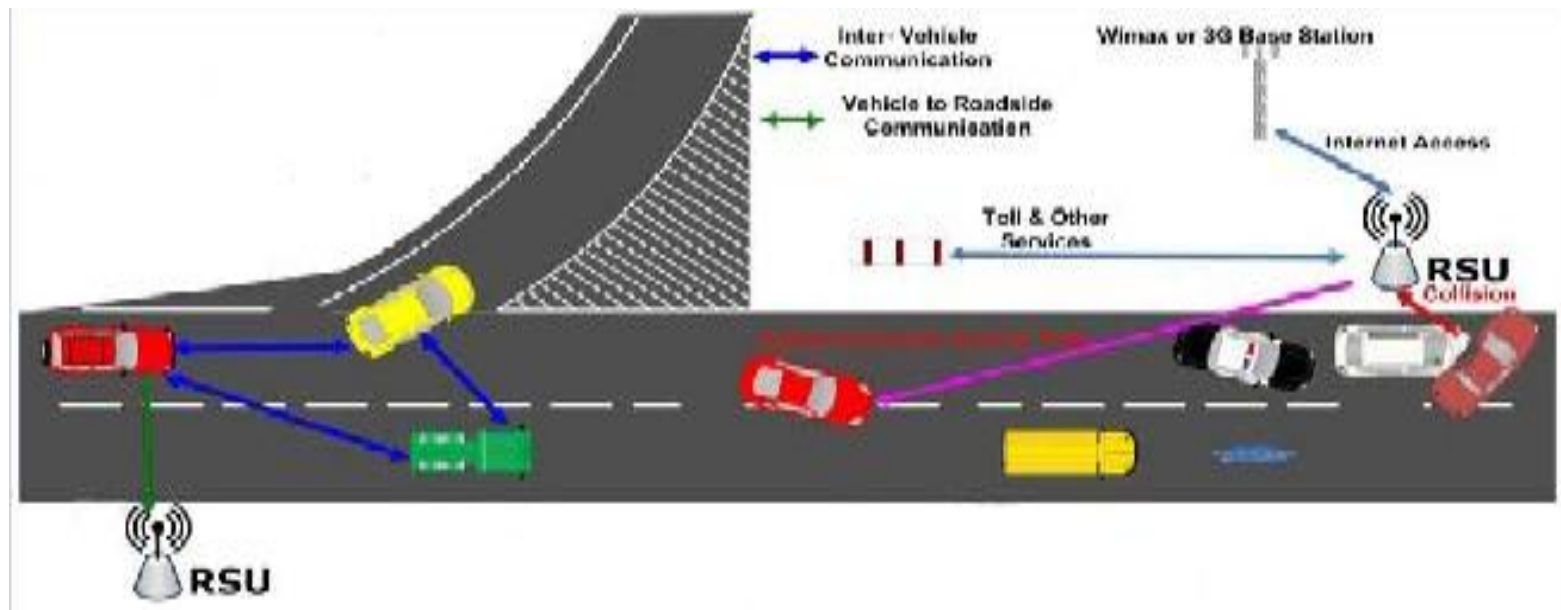

Figure8. Scenario depicting working of Geocast routing protocols 


\section{Challenges in Vanets}

\section{Quality of Service (QoS)}

QoS can be provided by the network with a minimum delay for data delivery, minimum retransmissions and high connectivity time. Offering this kind of dynamic network environment is a challenging task for VANETs.

\section{Efficient Routing Algorithm Design}

Designing an algorithm which can be implemented in different topologies of the network and satisfies conditions of minimum delay, maximum system capacity and decreased computational complexity, is another challenging task in VANETs.

\section{Scalability and Robustness}

Many complications are witnessed when traffic scenario changes dynamically form high mobility to slow movement. A complete framework that can adjust itself to such varying environment is required.

\section{$>$ Co-operative Communication}

VANETs works on the principle of communication between different nodes. The issue of settling to which extent nodes should exchange information among themselves is key research area in VANETs.

\section{Network Security}

Making sure that certain critical privacy information remains within the concerned node is also important to address. It requires implementing trust-based security protocol in VANET.

\section{Pros and Cons of Vanets from Organizational Point}

\section{Pros}

VANETs offers countless benefits to organizations of varying size. Automobile high-speed internet access enables transforming the vehicle's onboard computer from a nifty gadget to an efficient productivity tool and making any web technology virtually available in the vehicle. Although there remain few security concerns, this does not limit its potential as an effective tool. VANETs have the ability to convert the dead time (time wasted while waiting for something) to live time (time that is being used to complete tasks). A traveler can turn a traffic jam to productive work time by accomplishing tasks like downloading emails, music or other related stuff. One can surf the internet and utilize time to its best. It would also permit free VoIP services like Skype or GoogleTalk and hence minimize telecommunications costs.

\section{Cons}

There's no doubt in the fact that the internet is a useful productive tool, provided if used cleverly and safely. But if utilized in an irresponsible manner, it can prove quite distracting, resulting in compromise on safety concerns. Like cell phones, the Internet can be alluring and distract users from the road. Surfing web, watching videos on YouTube can lead to dangerous accidents. While the Internet can be a useful productivity tool, it can also prove to be quite distracting, resulting in the safety and actually time-wasting concerns. Like cellular phones, the Internet can be enticing and can distract the user's concentration from the road. Practices like checking emails, surfing the web or even watching YouTube videos can engross drivers and lead to accidents. Often when drivers get the opportunity to do work while on road, they engage themselves in leisurely tasks like VoIP with family, listening to podcasts and watching news highlights.

\section{Conclusion}

In this paper, we discussed and analyzed different routing protocols available in VANETs. The paper concluded with discussing challenges that need to be addressed in the successful implementation of VANETs along with its pros and cons. Active research efforts need to be undertaken to bridge the gaps required to make VANETs a reality. 


\section{REFERENCES}

[1] Sommer F., Dressler, "Progressing toward Realistic Mobility Models in VANET Simulations," IEEE Communications Magazine, November, pp. 132-137, 2008.

[2] Harri J., Filali F. and Bonnet C., "Mobility models for vehicular ad hoc networks: a survey and taxonomy," IEEE Communications Surveys \& Tutorials, vol. 11, no. 4, pp. 19-41, 2009.

[3] Dhurandher S.K., Obaidat M.S., Bhardwaj D., Garg A., "GROOV: A geographic routing over vanets and its performance evaluation", Global Communications Conference (GLOBECOM), 2012 IEEE, pp. 1670-1675.

[4] Kumar R., Dave M., "A comparative study of various routing protocols in Vanet," CoRR, vol. abs/1108.2094, 2011.

[5] Fonseca A., Vazo T., "Applicability of position-based routing for \{VANET \} in highways and urban environment," Journal of Network and Computer Applications, vol. 36, no. 3, pp. 961 973, 2013.

[6] Li B., Liu Y., Chu G., "Improved aodv routing protocol for vehicular ad hoc networks," 3rd International Conference on Advanced Computer Theory and Engineering (ICACTE), 2010, vol. 4, 2010, pp. V4-337-V4-340.

[7] Bhol, S.K.; Khillar, P.M., "A secure routing protocol for Vehicular Ad Hoc Network to provide ITS services," International Conference on Communication and Signal Processing (ICCSP), 2013, pp.117-124.

\section{AUTHOR's BIOGRAPHY}

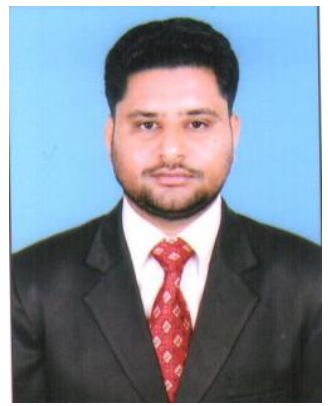

Dr. Gagandeep Jagdev, is a faculty member in Dept. of Computer Science, Punjabi University Guru Kashi College, Damdama Sahib (PB). His total teaching experience is above 10 years and has above 108 international and national publications in reputed journals and conferences to his credit. He is also a member of editorial board of several international peer-reviewed journals and has been active Technical Program Committee member of several international and national conferences conducted by renowned universities and academic institutions. His field of expertise is Big Data, ANN, Biometrics, RFID, Cloud Computing, Cryptography, and VANETS. 